\title{
CD39: the potential target in small cell lung cancer
}

\author{
Shanhao Chen ${ }^{1 \#}$, Shengyu Wu ${ }^{2,3 \#}$, Liping Zhang ${ }^{4}$, Wei Zhang ${ }^{4}$, Yu Liu ${ }^{2,3}$, Bin Chen ${ }^{2}$, Sha Zhao ${ }^{2}, \mathrm{Wei} \mathrm{Li}^{2}$, \\ Chenglong Sun ${ }^{2,5}$, Lei Wang ${ }^{2}$, Keyi Jia ${ }^{2,3}$, Hao Wang ${ }^{2,3}$, Peixin Chen ${ }^{2,3}$, Chunyan Wu ${ }^{4}$, Junjie Zhu ${ }^{6}$, \\ Yayi $\mathrm{He}^{2}$, Caicun Zhou ${ }^{2}$
}

${ }^{1}$ Medical College of Soochow University, Suzhou, China; ${ }^{2}$ Department of Medical Oncology, Shanghai Pulmonary Hospital, Tongji University Medical School Cancer Institute, Tongji University School of Medicine, Shanghai, China; ${ }^{3}$ Medical School, Tongji University, Shanghai, China; ${ }^{4}$ Pathology Department, Shanghai Pulmonary Hospital, Tongji University Medical School Cancer Institute, Tongji University School of Medicine, Shanghai, China; ${ }^{5}$ Anhui No.2 Provincial People's Hospital, Hefei, China; ${ }^{6}$ Department of Thoracic Surgery, Shanghai Pulmonary Hospital, Shanghai, China

Contributions: (I) Conception and design: C Zhou, Y He; (II) Administrative support: C Zhou, Y He; (III) Provision of study materials or patients: B Chen, S Zhao, W Li, C Sun, L Wang; (IV) Collection and assembly of data: L Zhang, C Wu; (V) Data analysis and interpretation: S Chen, S Wu, L Zhang, W Zhang, Y Liu, K Jia, H Wang, P Chen; (VI) Manuscript writing: All authors; (VII) Final approval of manuscript: All authors.

\#These authors contributed equally to this work.

Correspondence to: Caicun Zhou. Department of Medical Oncology, Shanghai Pulmonary Hospital, Tongji University Medical School Cancer Institute, Tongji University School of Medicine, No. 507 Zhengmin Road, Shanghai 200433, China; Email: caicunzhoudr@163.com; Yayi He. Department of Medical Oncology, Shanghai Pulmonary Hospital, Tongji University Medical School Cancer Institute, Tongji University School of Medicine, No. 507 Zhengmin Road, Shanghai 200433, China. Email: 2250601@qq.com.

Background: It has been proven that the treatment window of small cell lung cancer (SCLC) is short, so it is vital to find other possible therapeutic targets. CD39 inhibits natural killer (NK) cells and promotes the occurrence and metastasis of tumors. There has been little research about the role of CD39 in SCLC, so we explored the correlation between CD39 and other surface antigens, and its association with survival in SCLC.

Methods: This study included 75 patients with SCLC from Shanghai Pulmonary Hospital. After paraffin embedding and sectioning, immunohistochemistry (IHC) was applied. Then we identify cutoff value for CD39 and other surface antigens based on the analysis of ROC curve in RFS by SPSS. All statistical analyses were based on SPSS and Graphpad Prism8. Chi-square test, Kendall's tau-b correlation analysis, Logistic regression analysis, Kaplan-Meier method, univariate and multivariate Cox regression analysis were conducted. In all analyses, $\mathrm{P}=0.05$ distinguished whether they had statistical significance.

Results: Of the 75 SCLC patients enrolled in this study, 61.33\% positively expressed CD39. A correlation between CD39 and programmed cell death-ligand 1 ( $\mathrm{PD}-\mathrm{L} 1)(\mathrm{P}=0.007), \mathrm{CD} 3(\mathrm{P}<0.001), \mathrm{CD} 4(\mathrm{P}<0.001)$, CD8 $(\mathrm{P}<0.001)$, and forkhead box P3 (FOXP3) $(\mathrm{P}<0.001)$ on tumor-infiltrating lymphocytes (TILs) was identified by correlation analysis and logistic regression analysis. Based on Kaplan-Meier survival analysis, we found that CD39 affected relapse-free survival (RFS) [negative $v s$. positive, $95 \%$ confidence interval (CI): 0.2765-0.9862, $\mathrm{P}=0.0390]$. SCLC patients with high-expressed CD39 and low-expressed PD-L1 had poor prognosis $(\mathrm{P}<0.001)$. Positive expression of $\mathrm{CD} 39$ and negative expression of CD3, CD4, CD8, and FOXP3 also indicated shorter RFS ( $\mathrm{P}=0.0409)$. Univariate and multivariate Cox regression analysis was performed to confirm the factors that influenced RFS.

Conclusions: CD39, programmed cell death-1 (PD-1), and PD-L1 expressed on TILs but not on tumor cells. CD39 has a significant association with PD-L1, CD3, CD4, CD8, and FOXP3 on TILs. The positive expression of CD39 predicts poor prognosis. SCLC patients with low expression of CD39 combined with high expression of PD-L1 or CD3, CD4, CD8, and FOXP3 have a more favorable prognosis.

Keywords: CD39; small cell lung cancer (SCLC); programmed cell death-ligand 1 (PD-L1); tumor-infiltrating lymphocyte (TIL); progress-free survival; programmed cell death-1 (PD-1) 
Submitted Jun 04, 2020. Accepted for publication Jul 29, 2020.

doi: $10.21037 /$ tlcr-20-798

View this article at: http://dx.doi.org/10.21037/tlcr-20-798

\section{Introduction}

Small cell lung cancer (SCLC) is a pathological type of lung cancer, accounting for about $15 \%$ of lung cancer cases $(1,2)$. It originates from the precursors of neuroendocrine cells (3). SCLC proliferates, and incidence and mortality rates are high (4). The standard treatment for extensivestage SCLC (ES-SCLC) is 4 to 6 cycles of platinumbased double chemotherapy (5). Plenty of clinical trials mainly focused on ES-SCLC like CheckMate 032 (6), ECOG-ACRIN 2511 (7), CASPIAN (8) are currently undergoing. The therapeutic schedule in common use is surgical management for limited-stage SCLC (LS-SCLC) and chemotherapy and radiotherapy for ES-SCLC (9). Several studies have suggested that SCLC is sensitive to chemotherapy (10), yet the effect is temporary (11). Most patients are responsive to the initial treatment of platinum doublet therapy, but the majority with this metastatic disease still have rapid tumor progression $(12,13)$ It is of great importance to explore the function of other possible therapeutic targets.

The cluster of differentiation 39 (CD39), also known as ectonucleoside triphosphate diphosphohydrolase 1 (ENTPD1), can hydrolyze extracellular ATP and ADP (14). It is one of the key enzymes in adenosine pathway (15) and targeted adenosine can play a role in tumor immunotherapy (16). Tumour-derived exosomes (TDEs) change tumor microenvironment through the expression of CD39, which may be of great significance for immunotherapy as well (17). CD39 pathway inhibits natural killer (NK) cells and promotes the occurrence and metastasis of tumors (18) . Evidence shows it may evaluate the immunotherapy effect because it distinguishes tumorrelated or unrelated CD8 + tumor-infiltrating lymphocytes (TILs) in human solid tumors and causes the failure of CD8 + TILs (19-22). TGF- $\beta$-mTOR-HIF-1 signaling transduction is a pathway of adenosine pathway and up regulates CD39 (23). This demonstrates a possible signal transduction in NSCLC. Notably, it has previously shown that CD39 is highly elevated in intratumoral immune cells in NSCLC (24). Thus, CD39 can be viewed as a novel target for chemotherapy and immunotherapy in non-small cell lung cancer (NSCLC) (25).
Based on potential function and minimal existing research about CD39 in SCLC, we aimed to explore the correlation between CD39 and other surface antigens, including programmed cell death-ligand 1 (PD-L1), programmed cell death-1 (PD-1), CD3, CD4, CD8, and forkhead box P3 (FOXP3). We also researched whether it affected relapse-free survival (RFS) to assess its association with survival in SCLC.

Since Steven Rosenberg and his team found that CD39 may be a possible biomarker for advanced solid tumors (19), CD39 has offered a new strategy to treat advanced cancer patients. Although there were some articles about the relationship between CD39 and NSCLC in recent years, our study is the first to research in SCLC. Compared with NSCLC, malignant degree of SCLC is higher and it is easier to metastasis in early stage. Despite the sensitivity to chemotherapy, drug resistance is the main reason for poor prognosis (26). Considering the bad curative effect, it is much more important to find a potential target in SCLC. Additionally, we discovered the relationship between CD39 and other surface antigens in SCLC. Before this no one had done so. Advance experimental technology and means like immunohistochemistry were applied. Importantly, compared with others, various statistical methods such as univariate and multivariate Cox regression analysis were also used in this research to ensure the reliability of the experimental results. Thus, at current time, we make a break though research on CD39 in SCLC.

\section{Methods}

\section{Sample extraction}

This research was approved by the ethics committee of the Shanghai Pulmonary Hospital, Tongji University. Tumor samples were surgically collected from 75 patients with SCLC from Shanghai Pulmonary Hospital (from January 2014 to December 2018). Because of the small number of SCLC patients, we have included all SCLC patients in our hospital during this period to increase the amount of cases. Before the surgery, 46 patients had undergone chemotherapy, and 29 had not. The $8^{\text {th }}$ edition of the TNM classification for lung cancer was used to identify the 
varying stages of SCLC and prognosis of the patients (27). Written consent was given by all patients, and the experiment confirmed with the tenets of the Declaration of Helsinki (as revised in 2013).

\section{Paraffin embedding and sectioning}

Fresh tissues from the patients were fixed in $10 \%$ formalin for at least 24 hours (h). After placing them in the dehydration box, they were sequentially immersed in the following solutions for dehydration: $75 \%$ alcohol for $4 \mathrm{~h}$, $85 \%$ alcohol for 2 h, $90 \%$ alcohol for 2 h, $95 \%$ alcohol for $1 \mathrm{~h}$, anhydrous ethanol for 30 minutes ( $\mathrm{min}$ ), another anhydrous ethanol box for $30 \mathrm{~min}$, alcohol benzene for 5 $10 \mathrm{~min}$, xylene I for 5-10 min, xylene II for 5-10 minutes, wax I for $1 \mathrm{~h}$, wax II for $1 \mathrm{~h}$, and finally wax III for $1 \mathrm{~h}$. Next, we embedded the tissues in paraffin wax and cooled them at $-20{ }^{\circ} \mathrm{C}$ until they became wax blocks. After this entire process, we sliced the wax blocks to a thickness of $4 \mu \mathrm{m}$ and dried them (28).

\section{Immunohistochemistry (IHC) for CD39 and other surface antigens}

The prepared sections were first conventionally dewaxed to water. Antigen thermal remediation was performed by soaking the sections in phosphate buffer saline (PBS) and placing them in a $60{ }^{\circ} \mathrm{C}$ oven for $1 \mathrm{~h}$. Based on antigen repair, the sections were cooled at room temperature. The cooled sections were immersed in water for $2 \mathrm{~min}$ and then placed in $0.3 \% \mathrm{H}_{2} \mathrm{O}_{2}$ (an endogenous peroxidase inhibitor) for $15 \mathrm{~min}$ to reduce nonspecific background reactivity caused by endogenous peroxidase. Later, they were washed with water for $2 \mathrm{~min}$ and PBS buffer for $2 \mathrm{~min}$. To reduces nonspecific background staining, we next added Ultra $\mathrm{V}$ Block and incubated at room temperature for 5 min. Further, primary antibodies like CD39, PD-1 (1:100, Golden bridge Zhongshan, Beijing ZM-0381), PDL1 (E1L3N 1: 300, CST \# 13684S), CD3 (1:100, Dako A0452), CD4 (1:80, Dako M7310), CD8 (1:100, Dako M7103), FOXP3 (1:100, BioLegend 320101) were applied. After incubating at $37^{\circ} \mathrm{C}$ for $1-2 \mathrm{~h}$ and washing in a PBS buffer, we incubated an anti-enhancer at room temperature for $20 \mathrm{~min}$, washed with a PBS buffer, and added horseradish peroxidase (HRP) polymer (an enzyme-labeled

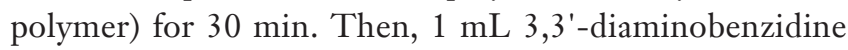
$(\mathrm{DAB})$ plus substrate and chromogen solution was added to each section. These sections were stained with hematoxylin and bluing agent. Finally, we started dehydration with $85 \%$ ethanol, $95 \%$ ethanol, and anhydrous ethanol. Xylene was used for transparency, and neutral gum was used as a sealant.

\section{The identification of cut off value for CD39 and other surface antigens}

TILs were composed of plasma cells, macrophages and lymphocytes (29). They were lymphocytes that leave the blood and migrate to the tumor area (30). We observed the lymphocytes in the microscope field of HE staining section mentioned above. $25 \%$ was selected as the cut off value of CD39 on TILs considering both sensitivity and specificity; this was decided after analysis of the ROC curve for RFS by IBM SPSS Statistics 22.0 (31). The cut-off value is calculated as the point which makes (sensitivity + specificity-1) maximum. According to the article published, the cut-off values for PD-1 and PD-L1 on tumor cells were $8 \%$ and $50 \%$, while PD- 1 and PD-L1 on TILs were $1 \%$ and $5 \%$ (32). Furthermore, CD3 >40\%, CD $4>30 \%$, CD 8 $>30 \%$ and FOXP $3>10 \%$ in staining were also viewed as positive in the same way.

\section{Statistics analysis}

All statistical analyses were based on SPSS (version 22.0) (31) and Graphpad Prism8 (33). We applied the Chi-square test to assess if the expression of CD39 on TILs and other clinicopathologic parameters were cognate. Kendall's tau-b correlation analysis was used for the evaluation of correlation and agreement of CD39 and other surface antigens. Logistic regression analysis was used to evaluate the effect of surface antigens. The Kaplan-Meier method was used to estimate the survival curves.

Additionally, univariate and multivariate Cox regression analysis was conducted to discover possible factors associated with prognosis. The odds ratio (OR) in the model referred to the correlation between surface antigens and RFS. In all analyses, $\mathrm{P}=0.05$ distinguished whether there was statistical significance.

\section{Results}

\section{Characteristics of patients enrolled in the study}

Out of the 75 SCLC patients included in our research, 62 were male and 13 female. The median age was 63 (upper 
Table 1 Baseline information of 75 patients diagnosed with SCLC

\begin{tabular}{|c|c|}
\hline Variables & Total patients $(\mathrm{N}=75)$ \\
\hline \multicolumn{2}{|l|}{ Age, years } \\
\hline Mean \pm SD & $63.28 \pm 9.46$ \\
\hline Median (range) & $63(38-81)$ \\
\hline \multicolumn{2}{|l|}{ Gender } \\
\hline Female & 13 (17.33\%) \\
\hline Male & $62(82.67 \%)$ \\
\hline \multicolumn{2}{|l|}{ Smoking status } \\
\hline Non-smoker & 32 (42.67\%) \\
\hline Smoker & 43 (57.33\%) \\
\hline \multicolumn{2}{|l|}{ SCLC stage } \\
\hline Stage I & 30 (40.00\%) \\
\hline Stage II & $10(13.33 \%)$ \\
\hline Stage III & 35 (46.67\%) \\
\hline \multicolumn{2}{|l|}{$\mathrm{T}$} \\
\hline $\mathrm{T} 1$ & 32 (42.67\%) \\
\hline $\mathrm{T} 2$ & $31(41.33 \%)$ \\
\hline T3 & $10(13.33 \%)$ \\
\hline T4 & $2(2.67 \%)$ \\
\hline \multicolumn{2}{|l|}{$\mathrm{M}$} \\
\hline Mo & 72 (96.0\%) \\
\hline M1 & $3(4.00 \%)$ \\
\hline \multicolumn{2}{|l|}{$\mathrm{N}$} \\
\hline No & 34 (45.33\%) \\
\hline $\mathrm{N} 1$ & $13(17.33 \%)$ \\
\hline N2 & 28 (37.34\%) \\
\hline \multicolumn{2}{|l|}{ Chemotherapy } \\
\hline Yes & $46(61.33 \%)$ \\
\hline No & 29 (38.67\%) \\
\hline
\end{tabular}

SCLC, small cell lung cancer.

limit: 81 , lower limit: 38$)$. The majority (57.33\%) were smokers. By TNM classification from The IASLC Lung Cancer Staging Project $8^{\text {th }}$ Version (27), all patients were sorted into T1-T4, M0-M1, and N0-N2. The baseline information of all patients enrolled is displayed in Table 1.

\section{Expression of surface antigens on different cells tested by IHC}

After IHC, we performed qualitative and relative quantitative research on the surface antigens. In this study, CD39, PD-1, and PD-L1 have no expression on tumor cells; $61.33 \%$ of patients expressed CD39 positively on TILs while $38.67 \%$ did not. The positive expression on TILs was $38.67 \%$ for PD- 1 and $37.33 \%$ for PD-L1. The number of SCLC patients who expressed CD3, CD4, CD8, and FOXP3 is displayed in Table 2.

\section{Exploration for the relationship of surface antigens and clinical data}

We set $\mathrm{P}=0.05$ as a threshold. By Chi-square test, the expression of CD39 on TILs had no statistical correlation with clinical data like age $(\mathrm{P}=0.428)$, gender $(\mathrm{P}=0.549)$, smoking status $(\mathrm{P}=0.477)$, SCLC stage $(\mathrm{P}=1.000)$, and chemotherapy $(\mathrm{P}=0.336)$ (Table 3). Meanwhile, a possible correlation between CD39 expression on TILs and some other surface antigens was proven with $\mathrm{P}<0.05$. More specifically, PD-L1 ( $\mathrm{P}=0.007), \mathrm{CD} 3(\mathrm{P}<0.001)$, CD4 $(\mathrm{P}<0.001)$, CD8 $(\mathrm{P}<0.001)$, and FOXP3 $(\mathrm{P}<0.001)$ expression on TILs were statistically correlated with CD39 expression on TILs (Table 3).

\section{Correlation analysis and logistic regression analysis}

Kendall's tau-b correlation analysis was performed. All possible statistically correlated surface antigens mentioned above were included in the calculation to confirm a significant correlation. The specific Kendall's tau-b and p-value of the targets are shown in Table 4. Results showed that the expression of PD-L1, CD3, CD4, CD8, and FOXP3 on TILs had a positive correlation with CD39 expression on TILs. This result had a statistical sense. Among all of them, the CD3 expression had the highest relevancy (Kendall's tau- $\mathrm{b}=0.552, \mathrm{P}<0.001$ ).

Based on bivariate logistic regression, we once again supported the statistical correlation mentioned above as $\mathrm{P} \leq 0.05$ (Figure 1, Table S1). All regression coefficients were more significant than zero, which means a positive correlation coefficient between variables. CD8 expression had the maximum regression coefficient of 3.183 but had no statistical significance $(\mathrm{P}=0.998)$. Bivariate logistic 
Table 2 Relationships between CD39 expression on TILs and other checkpoints (Chi-square test)

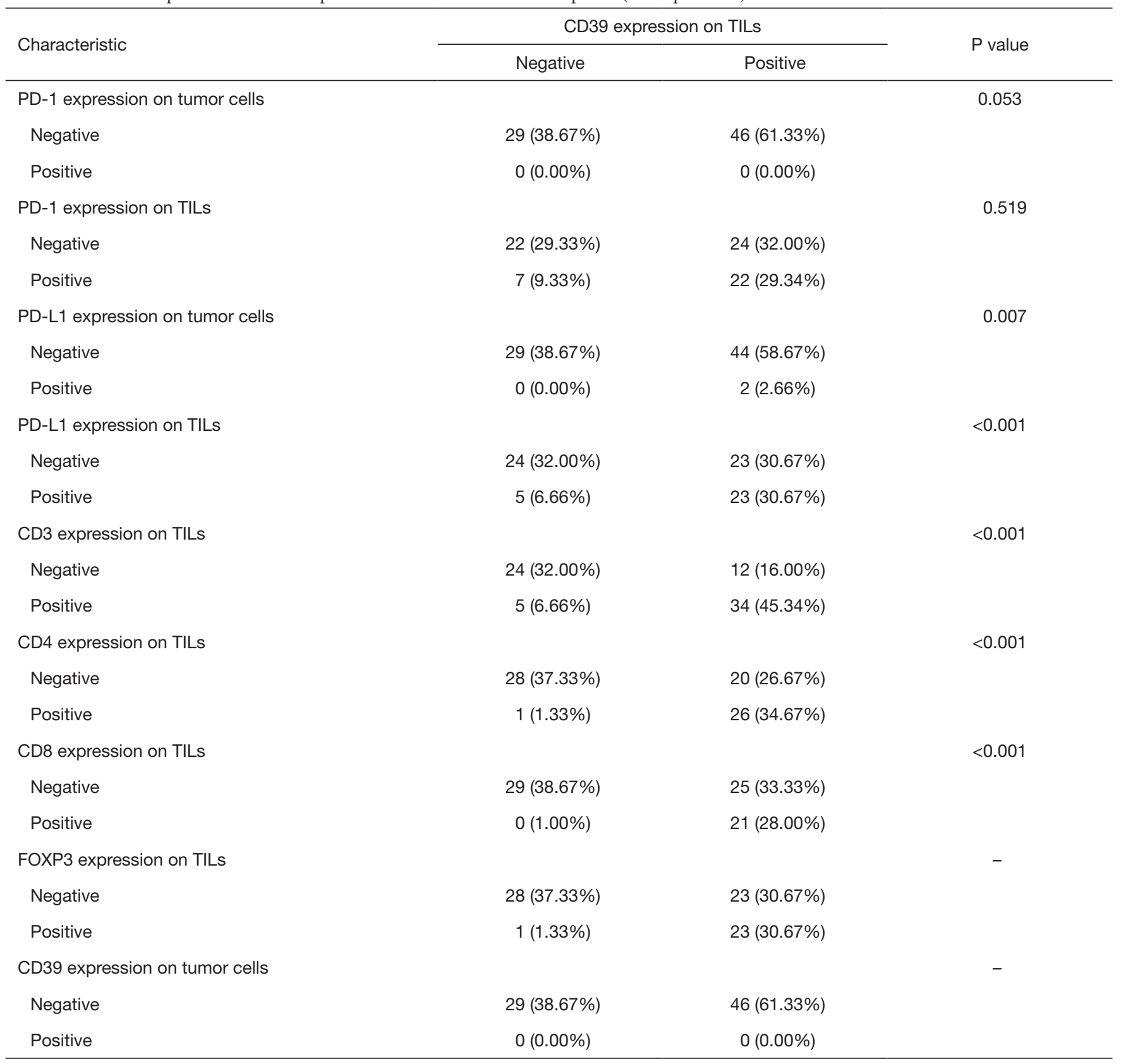

TIL, tumor-infiltrating lymphocyte.

regression of PD-L1, CD3, CD4, and FOXP3 on TILs showed that CD39 impacted them greatly.

In summary, the significant association between CD39 expression on TILs, and PD-L1, CD3, CD4, and FOXP3 was identified based on correlation analysis and logistic regression analysis performed after the Chi-square test.

\section{Predictive factors of RFS in SCLC}

By Kaplan-Meier survival analysis, relationships between RFS status and clinical data were evaluated (Figure $2 A, B, C, D, E$, Table S2). It was confirmed that age (<70 vs. $>70,95 \%$ CI: $0.1964-0.9172, \mathrm{P}=0.0119)$ and SCLC stage (Stage I-II vs. Stage III-IV, 95\% CI: $0.2186-$ 
Table 3 Relationships between CD39 and clinical data

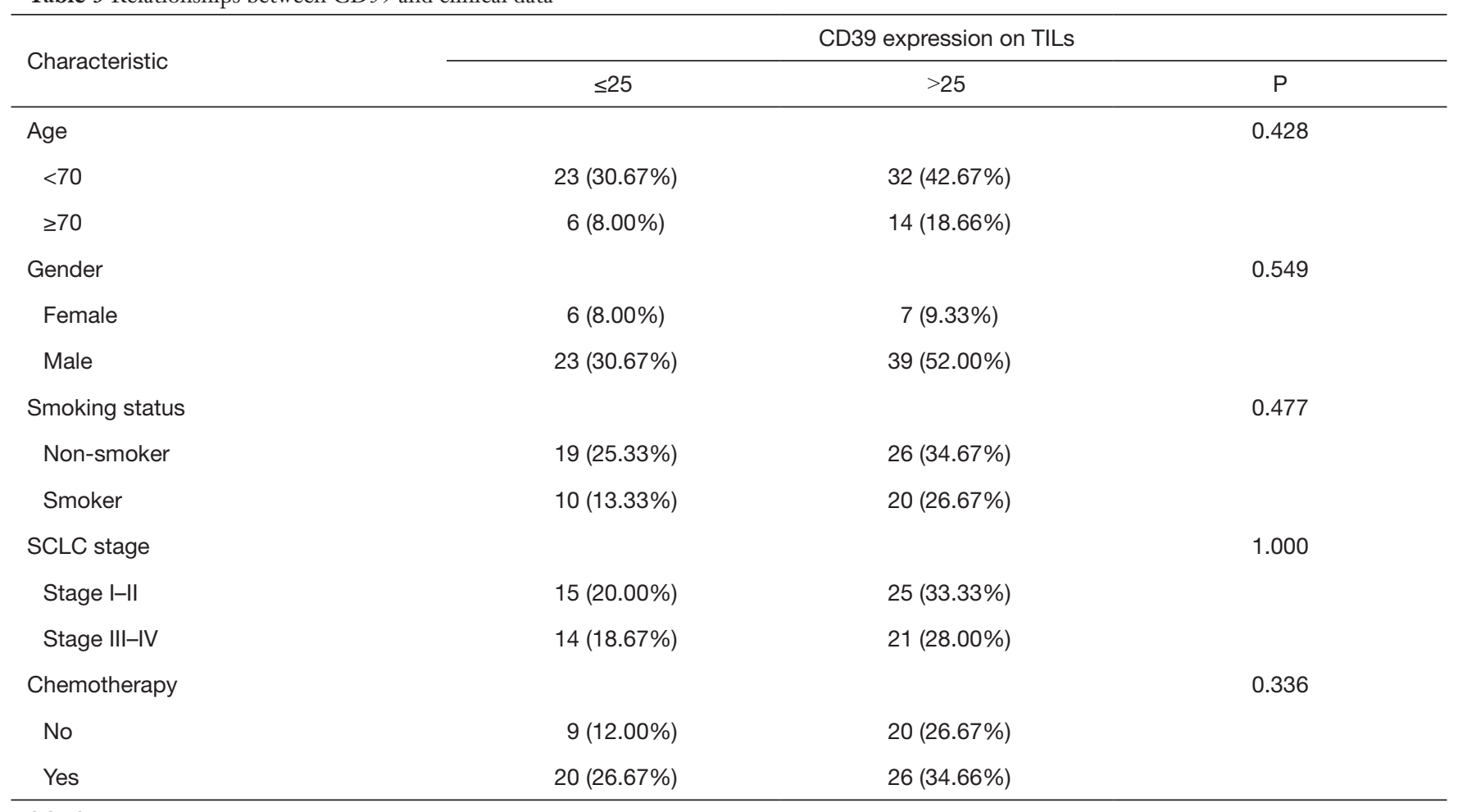

SCLC, small cell lung cancer.

Table 4 Relationships between CD39 expression on TILs and other checkpoints (Kendall's tau-b correlation analysis)

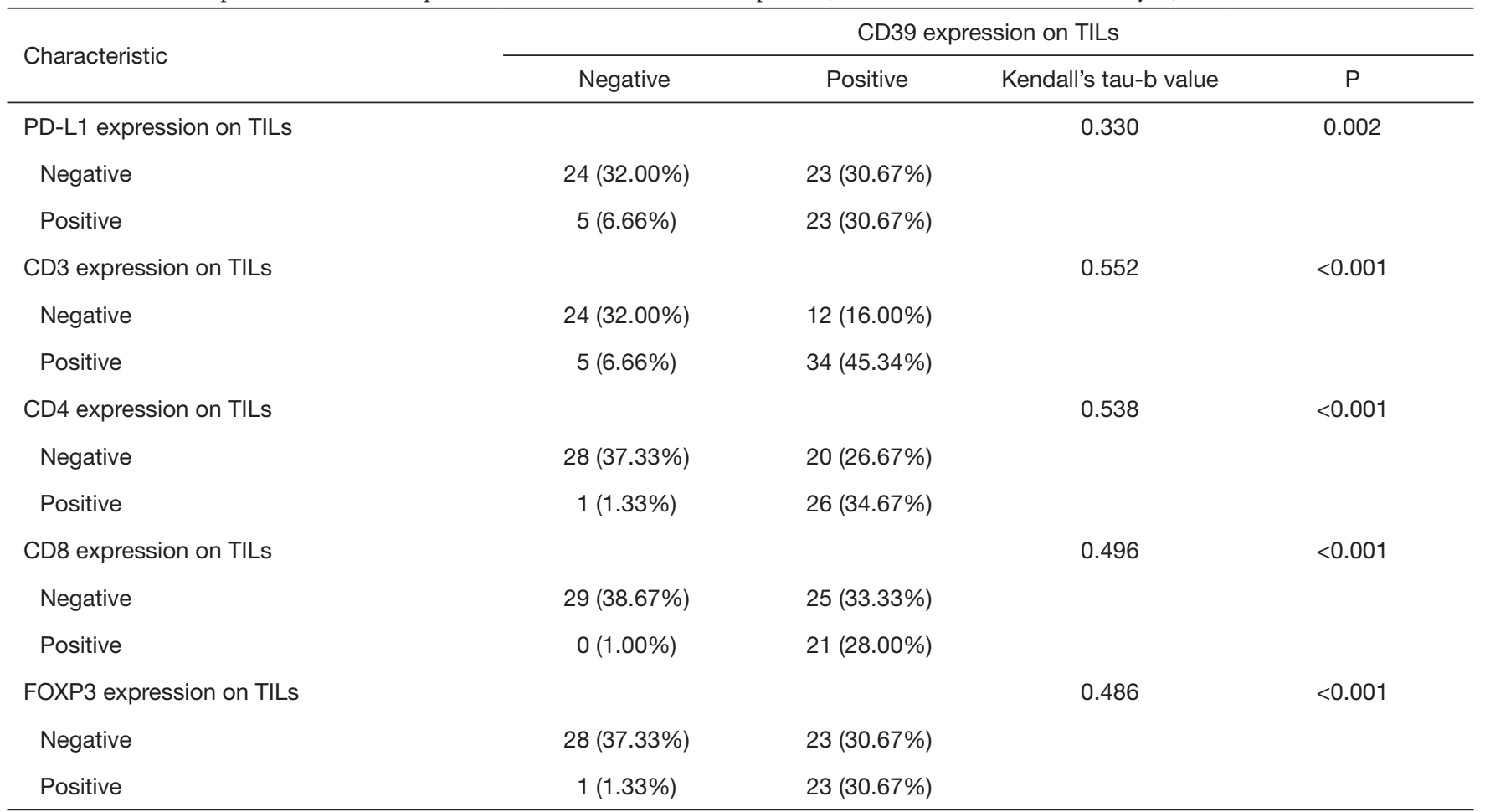

PD-L1, programmed cell death-ligand 1; FOXP3: forkhead box P3; TIL, tumor-infiltrating lymphocyte. 


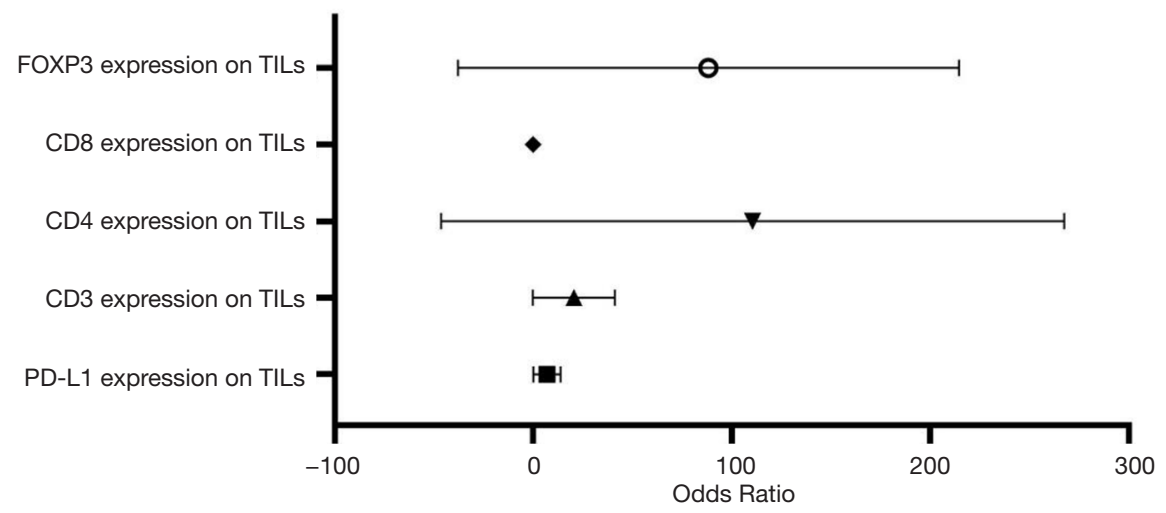

Figure 1 Logistic regression analysis. Bivariate logistic regression of PD-L1, CD3, CD4, CD8, and FOXP3. FOXP3, forkhead box P3; TIL, tumor-infiltrating lymphocyte; PD-L1, programmed cell death-ligand 1.
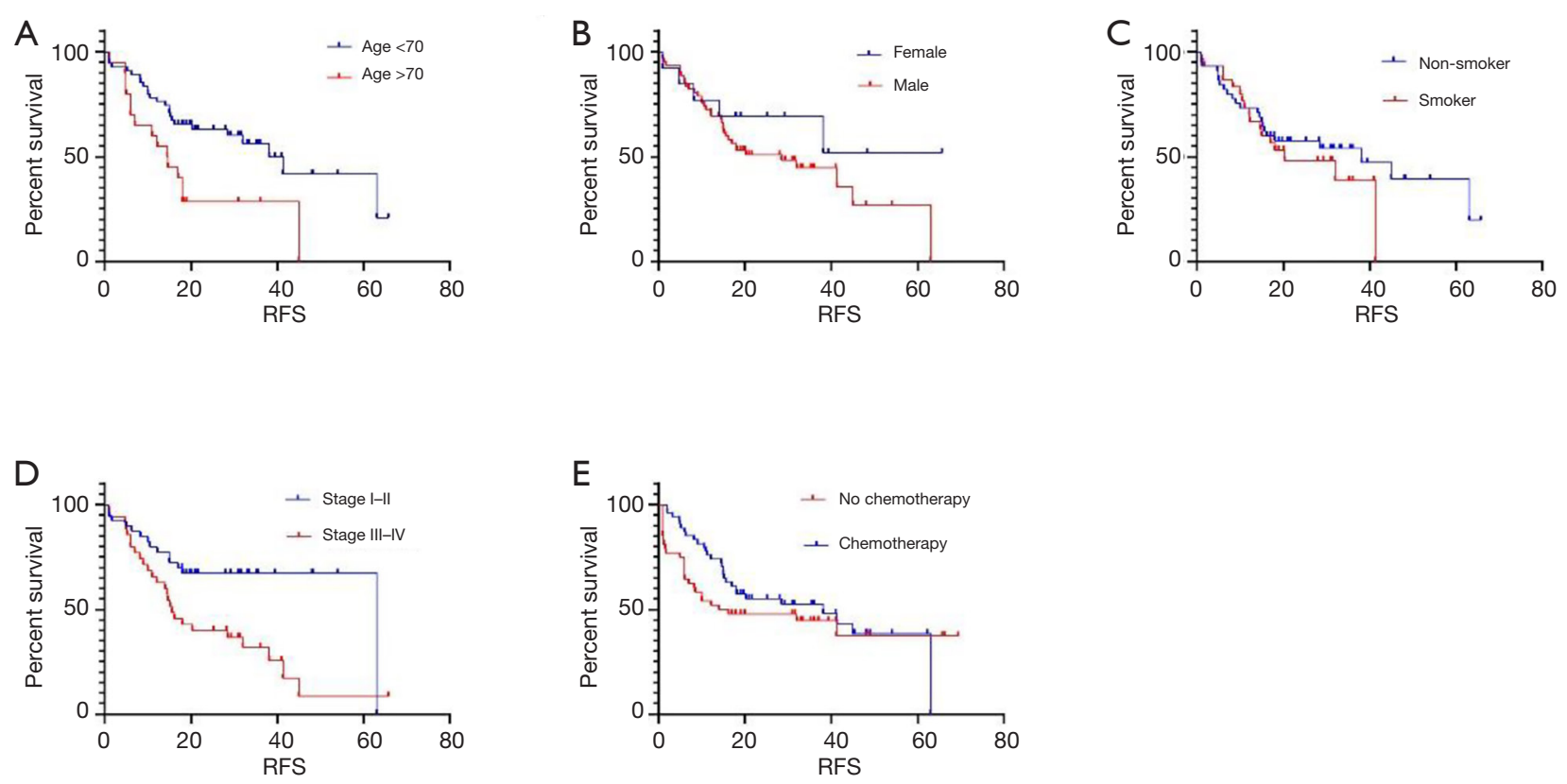

Figure 2 Kaplan-Meier curve for clinical factors related to prognosis. Kaplan-Meier curve for age (A), gender (B), smoking (C), SCLC stage (D), chemotherapy (E). RFS, relapse-free survival; SCLC, small cell lung cancer.

0.7640, $(\mathrm{P}=0.0050)$ were significantly associated with RFS, while gender $(\mathrm{P}=0.2318)$, smoker status $(\mathrm{P}=0.4255)$ and chemotherapy $(\mathrm{P}=0.3256)$ had no statistical associations. Age and SCLC stages were considered as risk factors, as hazard ratios (HR) were less than one ( $<70 v s .>70$, Stage III vs. Stage III-IV).

The association between RFS and surface antigens was also explored using the Kaplan-Meier method (Figure $3 A, B, C, D, E, F$, Table S2) and whether CD39 expressed on TILs or not significantly impacted RFS [negative vs. positive (neg vs. pos), 95\% CI: $0.2765-0.9862$, $\mathrm{P}=0.0390]$. The same was found to be true for PD-L1 (neg vs. pos, 95\% CI: 1.177-4.190, $\mathrm{P}=0.0256$ ), CD3 (neg vs. pos, 95\% CI: 1.037-3.868, $\mathrm{P}=0.0342$ ), $\mathrm{CD} 4$ (neg vs. pos, 95\% CI: 1.125-4.169, $\mathrm{P}=0.0367$ ), CD8 (neg vs. pos, 95\% CI: 1.136-4.597, $\mathrm{P}=0.0497$ ) and FOXP3 (neg vs. pos, 95\% CI: $1.137-4.374, \mathrm{P}=0.0440$ ) expression on TILs. In summary, CD39 was the risk factor, while PD-L1, CD3, CD4, CD8, and FOXP3 on TILs had a protective effect for SCLC patients. 

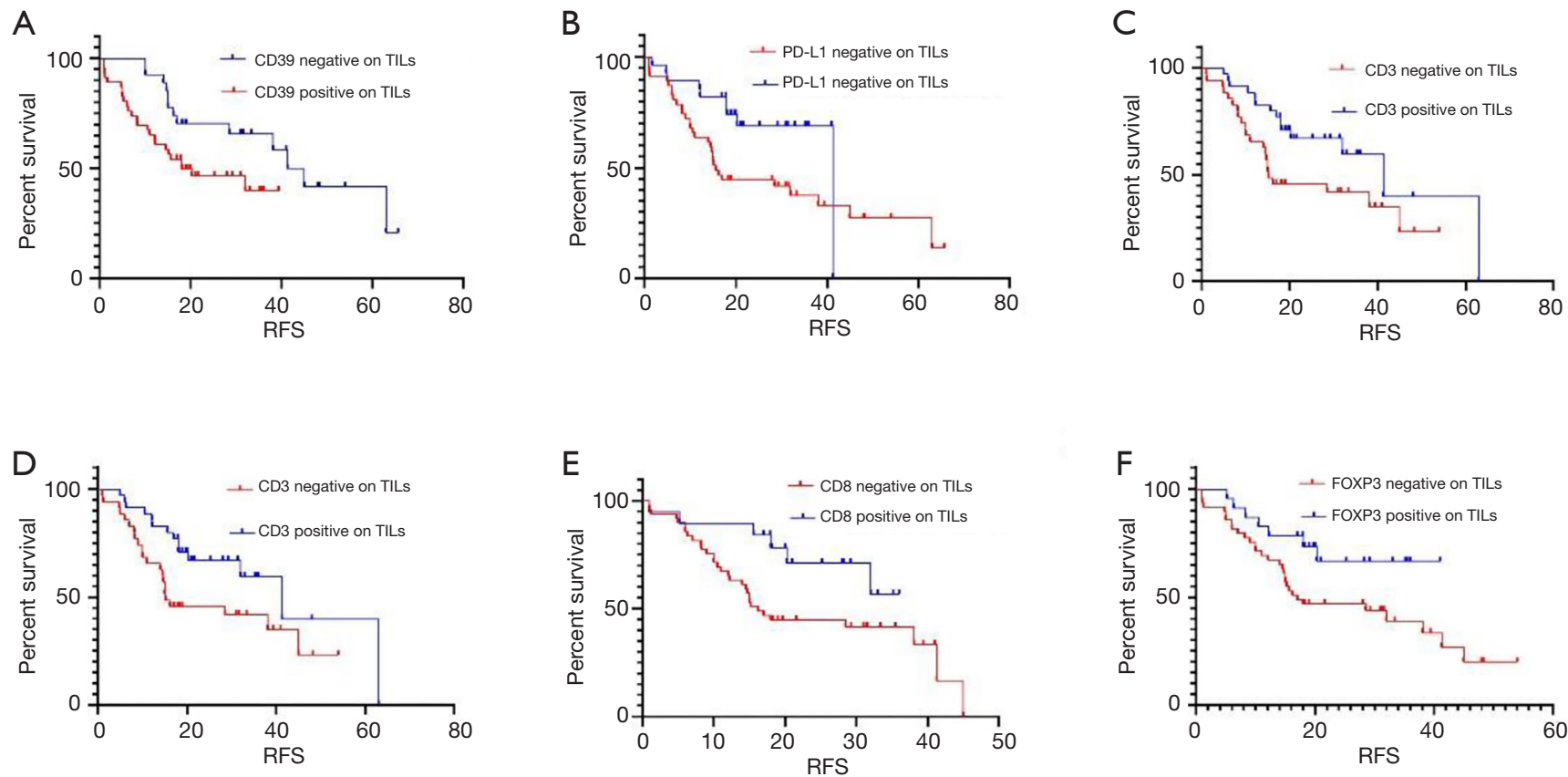

Figure 3 Kaplan-Meier curve for surface antigens. Kaplan-Meier curve for CD39 (A), PD-L1 (B), CD3 (C), CD4 (D), CD8 (E), FOXP3 (F). TIL, tumor-infiltrating lymphocyte; RFS, relapse-free survival; PD-L1, programmed cell death-ligand 1; FOXP3, forkhead box P3.
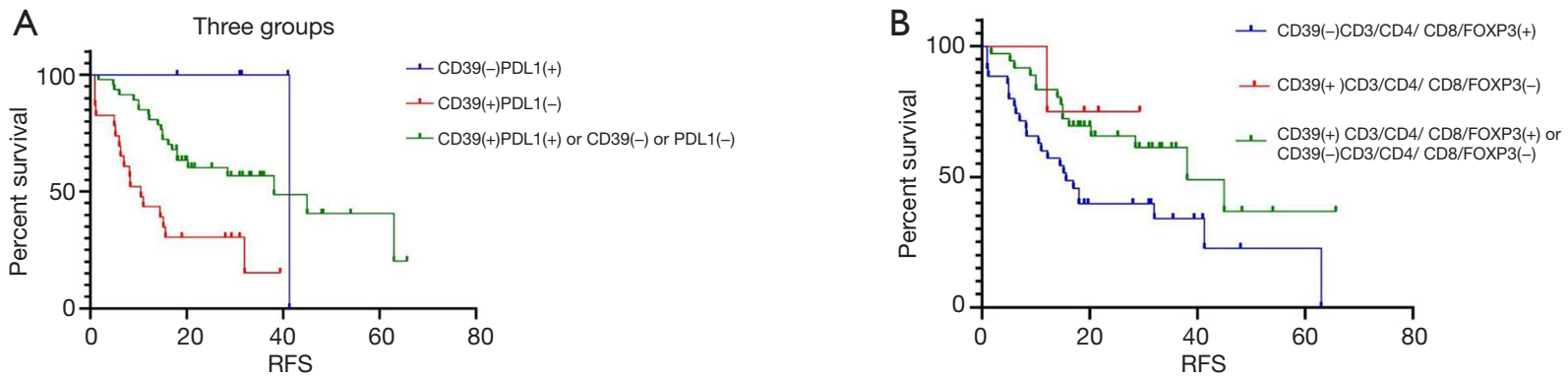

Figure 4 Kaplan-Meier curve for subgroups. (A) The expression of CD39 combined with PD-L1. (B) CD39 combined with CD3, CD4, CD8, and FOXP3. RFS, relapse-free survival; PD-L1, programmed cell death-ligand 1; FOXP3, forkhead box P3.

After evaluating each surface antigen separately, we created subgroups and combined them for analysis. Because $\mathrm{CD} 3, \mathrm{CD} 4, \mathrm{CD} 8$, and FOXP3 were the primary markers of lymphocytes, we finally divided all surface antigens mentioned above into three groups: CD39, PD-L1 on TILs, and primary markers of lymphocytes (CD3, CD4, CD8, and FOXP3). The result of Kaplan-Meier analysis was that SCLC patients with low expression of CD39 and high expression of PD-L1 had the best RFS, while the positive expression of CD39 and negative expression of $\mathrm{PD}-\mathrm{L} 1$ suggested poor prognosis $(\mathrm{P}=0.0007)$ (Figure $4 A$ ). Positive expression of CD39 and negative expression of
CD3, CD4, CD8, and FOXP3 also indicated shorter RFS and poorer prognosis $(\mathrm{P}=0.0409)$ (Figure $4 B$ ).

It was proven that $\mathrm{CD} 39, \mathrm{PD}-\mathrm{L} 1, \mathrm{CD} 3, \mathrm{CD} 4, \mathrm{CD} 8$, and FOXP3 are all involved in SCLC prognosis and improve patients' prognosis.

\section{Univariate and multivariate Cox regression analyses of RFS}

Univariate and multivariate Cox regression analyses were performed to confirm the possible factors that influenced RFS (Table S3). By using univariate Cox regression analysis, 


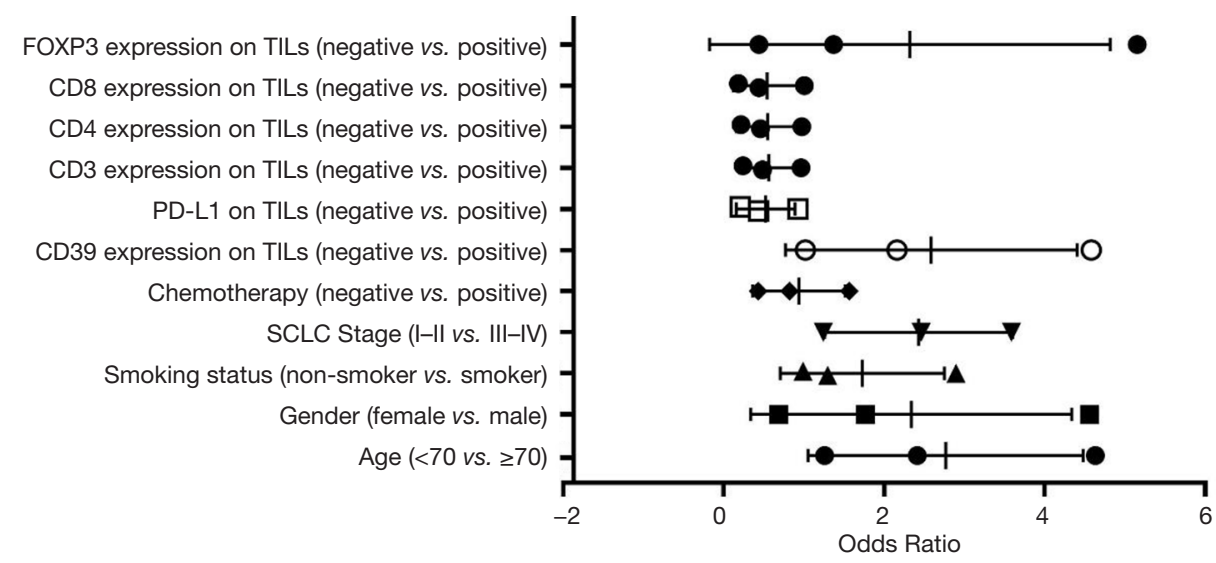

Figure 5 Univariate Cox regression analysis. Univariate Cox regression analysis of clinical factors and surface antigens with RFS. FOXP3, forkhead box P3; TIL, tumor-infiltrating lymphocyte; PD-L1, programmed cell death-ligand 1; SCLC, small cell lung cancer; RFS, relapsefree survival.

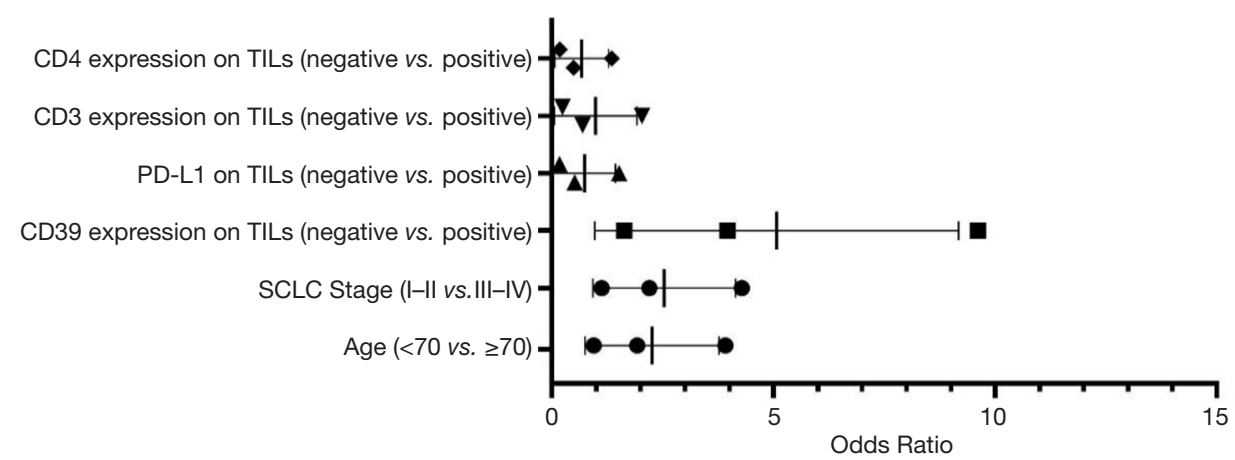

Figure 6 Multivariate Cox regression analysis. Multivariate Cox regression analysis of clinical factors and surface antigens with RFS. TIL, tumor-infiltrating lymphocyte; PD-L1, programmed cell death-ligand 1; SCLC, small cell lung cancer; RFS, relapse-free survival.

we found age $(\mathrm{P}=0.008)$, SCLC stage $(\mathrm{P}=0.007)$, CD39 ( $\mathrm{P}=0.044)$, PD-L1 $(\mathrm{P}=0.030)$, CD3 $(\mathrm{P}=0.039)$, and CD4 $(\mathrm{P}=0.042)$ expression on TILs had a significant association with RFS (Figure 5). Cox regression analysis was performed to eliminate confounding effects (Figure 6), and it demonstrated a significant correlation between the SCLC stage $(\mathrm{P}=0.021)$, CD39 expression on TILs $(\mathrm{P}=0.002)$, and RFS.

\section{Discussion}

SCLC progresses rapidly and has a poor prognosis (34-36). However, immunotherapy offers new prospects for SCLC patients, and findings of trials from recent years have supported this. The trial CheckMate-032 $(37,38)$ finds nivolumab monotherapy to be an effective third- line therapy (37). KEYNOTE-028 (39) reveals good treatment results using pembrolizumab for PD-L1positive SCLC (39). IMpower133 shows that atezolizumab combined with etoposide and carboplatin could prolong overall survival (OS) and progression-free survival (PFS) for SCLC patients (40), and CASPIAN proves the role of PD-L1 inhibitors combined with chemotherapy in improving OS (41). CD39 may have a significant role in the evaluation of the effect of immunotherapy because the differential expression of CD39 is key to distinguishing tumor-related or unrelated CD8 + TILs (19-22). It may be a potential target for immunotherapy in glioblastoma because of its function in tumor-related macrophages and T cells (42). CD39 blocking may work in the eATP-P2X7inflammasome-IL18 axis and decrease the number of macrophages in the tumor as a result (43). Little research 
has been done regarding the role of CD39 in NSCLC. Evidence shows that anti-CD39 combined with anti-PD-1 can inhibit the metastasis of NSCLC (44), and this may be related to inhibiting the $\mathrm{CD} 39 / \mathrm{CD} 73$-adenosine pathway, an essential mechanism of tumor immunosuppression (25). However, until now, no other study about the impact of CD39 on SCLC had been conducted. We are the first to explore the possible relationship between CD39 and SCLC and its connection with other surface antigens like PD-1, PD-L1, CD3, CD4, CD8, and FOXP3.

We obtained 75 clinical samples upon which IHC was performed, and the expression of surface antigens on different cells was expressed. There were $38.67 \%$ of patients expressed CD39 positively on TILs. Methods including the Chi-square test, Kendall's tau-b correlation analysis, and logistic regression analysis were used to explore and confirm the possible relationship between CD39 and other surface antigens. In SCLC, PD-L1 expression on TILs, CD3, CD4, and FOXP3 expression on cells was significantly correlated with CD39 expression on TILs. Ahlmanner et al. found the same situation in colon tumors: tumor-infiltrating CD39+ regulatory T cells (Tregs) high expressed immunosuppressive molecules like PD-L1 and cytotoxic t-lymphocyte associated protein 4 (CTLA-4) (45). A study by Syed Khaja also proved the coexpression of PD-1/CTLA-4 and PD-1/CD39, adding that the expression of CD4 in $\mathrm{T}$ cells constitutes the $\mathrm{CD} 3+\mathrm{T}$ cells in the tumor microenvironment (TME) and promotes tumor metastasis (46).

Using Kaplan-Meier survival analysis, we looked for factors that may be related to prognosis. Age and SCLC stage appear to be risk factors of SCLC, with HR $<1 \quad(<70$ $v s$. $>70$, Stage I-II vs. Stage III-IV). CD39 expression on TILs is another identified risk factor. This is consistent with Canale's study, where CD39 was viewed as an immunosuppressive molecule that induces CD8+ $\mathrm{T}$ cell exhaustion (47). This has also been proven in tumors of oral and gastric cancer (48), colorectal cancer (49), NSCLC $(25,50,51)$, and others. In our findings, PD-L1, CD3, CD4, CD8, and FOXP3 expression on TILs had a protective effect in SCLC patients. Among them, CD3 promotes a good prognosis for many cancers, including SCLC (52-54).

Because of the significant correlation between CD39 and other surface antigens, we divided the samples into subgroups and found SCLC patients with low expression of CD39 and high expression of PD-L1 had the best RFS while the positive expression of CD39 and negative expression of PD-L1 suggested poor prognosis. Positive expression of CD39 combined with negative expression of CD3, CD4, CD8, and FOXP3 also indicated shorter RFS and poorer prognosis.

In summary, we discovered the relationship between CD39 and other surface antigens in SCLC, along with the related risk and protective factors for RFS. Admittedly, our study had some limitations; most notable was the small sample size; all patients were recruited from the Shanghai Pulmonary Hospital. In order to reduce the error caused by small sample size, we divided subgroups to better understand the relationship between CD39 and SCLC, moreover, the significant correlation between CD39 and other surface antigens. We also used various statistical methods, for example, Chi-square test, Kendall's tau-b correlation analysis, Logistic regression analysis, KaplanMeier method, univariate and multivariate Cox regression analysis to reduce errors as much as possible. In spite of the limitations, our study was the first to analyze CD39 in SCLC and its relationship with PD-L1, CD3, CD4, CD8, and FOXP3. According to our findings, CD39 is a potential target for therapy. This needs to be verified through further animal and clinical research.

\section{Conclusions}

CD39 expresses on TILs and has a significant association with PD-L1 on TILs, CD3, CD4, CD8, and FOXP3. The positive expression of CD39 leads to short RFS and poor prognosis. Low expression of CD39 combined with high expression of PD-L1 or CD3, CD4, CD8, and FOXP3 indicates a good prognosis for SCLC patients.

\section{Acknowledgments}

Funding: This study was supported in part by a grant from the National Natural Science Foundation of China (81802255), Shanghai Pujiang Program (17PJD036), a grant from Shanghai Municipal Commission of Health and Family Planning Program (20174Y0131), National Key Research \& Development Project (2016YFC0902300), Major disease clinical skills enhancement program of the three-year action plan for promoting clinical skills and clinical innovation in municipal hospitals, Shanghai Shen Kang Hospital Development Center Clinical Research Plan of SHDC (16CR1001A), Dream Tutor Outstanding Young Talents Program (fkyq1901), Key Disciplines of Shanghai Pulmonary Hospital (2017ZZ02012), the grant of Shanghai Science and Technology Commission (16JC1405900). 


\section{Footnote}

Data Sharing Statement: Available at http://dx.doi. org/10.21037/tlcr-20-798

Conflicts of Interest: All authors have completed the ICMJE uniform disclosure form (available at http://dx.doi. org/10.21037/tlcr-20-798). The authors have no conflicts of interest to declare.

Ethical Statement: The authors are accountable for all aspects of the work in ensuring that questions related to the accuracy or integrity of any part of the work are appropriately investigated and resolved. This research was approved by the ethics committee of the Shanghai Pulmonary Hospital, Tongji University (No. K20022). Written consent was given by all patients, and the experiment confirmed with the tenets of the Declaration of Helsinki (as revised in 2013).

Open Access Statement: This is an Open Access article distributed in accordance with the Creative Commons Attribution-NonCommercial-NoDerivs 4.0 International License (CC BY-NC-ND 4.0), which permits the noncommercial replication and distribution of the article with the strict proviso that no changes or edits are made and the original work is properly cited (including links to both the formal publication through the relevant DOI and the license). See: https://creativecommons.org/licenses/by-nc-nd/4.0/.

\section{References}

1. Tsoukalas N, Aravantinou-Fatorou E, Baxevanos P, et al. Advanced small cell lung cancer (SCLC): new challenges and new expectations. Ann Transl Med 2018;6:145.

2. Sun $Y$, Zhai C, Chen X, et al. Characterization of PD$\mathrm{L} 1$ protein expression and CD8+ tumor-infiltrating lymphocyte density, and their associations with clinical outcome in small-cell lung cancer. Transl Lung Cancer Res 2019;8:748-59.

3. Govindan R, Page N, Morgensztern D, et al. Changing epidemiology of small-cell lung cancer in the United States over the last 30 years: analysis of the surveillance, epidemiologic, and end results database. J Clin Oncol 2006;24:4539-44.

4. Lee SY, Choi YJ, Seo JH, et al. Pulmonary function is implicated in the prognosis of metastatic non-small cell lung cancer but not in extended disease small cell lung cancer. J Thorac Dis 2019;11:4562-72.

5. Waqar SN, Morgensztern D. Treatment advances in small cell lung cancer (SCLC). Pharmacol Ther 2017;180:16-23.

6. Ready N, Farago AF, de Braud F, et al. Third-Line Nivolumab Monotherapy in Recurrent SCLC: CheckMate 032. J Thorac Oncol 2019;14:237-44.

7. Owonikoko TK, Dahlberg SE, Sica GL, et al. Randomized Phase II Trial of Cisplatin and Etoposide in Combination With Veliparib or Placebo for Extensive-Stage Small-Cell Lung Cancer: ECOG-ACRIN 2511 Study. J Clin Oncol 2019;37:222-9.

8. Paz-Ares L, Dvorkin M, Chen Y, et al. Durvalumab plus platinum-etoposide versus platinum-etoposide in firstline treatment of extensive-stage small-cell lung cancer (CASPIAN): a randomised, controlled, open-label, phase 3 trial. Lancet 2019;394:1929-39.

9. Chen P, Kuang P, Wang L, et al. Mechanisms of drugsresistance in small cell lung cancer: DNA-related, RNA-related, apoptosis-related, drug accumulation and metabolism procedure. Transl Lung Cancer Res 2020;9:768-86.

10. Gazdar AF, Bunn PA, Minna JD. Small-cell lung cancer: what we know, what we need to know and the path forward. Nat Rev Cancer 2017;17:725-37.

11. Roth BJ JD, Einhorn LH, et al. Randomized study of cyclophosphamide, doxorubicin, and vincristine versus etoposide and cisplatin versus alternation of these two regimens in extensive small-cell lung cancer: a phase III trial of the Southeastern Cancer Study Group. J Clin Oncol 1992;10:282-91.

12. Waqar SN, Morgensztern D. Treatment advances in small cell lung cancer (SCLC). Pharmacol Ther 2017;180:16-23.

13. Stinchcombe TE. Current Treatments for Surgically Resectable, Limited-Stage, and Extensive-Stage Small Cell Lung Cancer. Oncologist 2017;22:1510-7.

14. Mosaad Zaki E, Mohamed Zahran A, Abdelazeem Metwaly A, et al. Impact of CD39 expression on CD4+ T lymphocytes and 6q deletion on outcome of patients with chronic lymphocytic leukemia. Hematol Oncol Stem Cell Ther 2019;12:26-31.

15. Allard B, Longhi MS, Robson SC, et al. The ectonucleotidases CD39 and CD73: Novel checkpoint inhibitor targets. Immunol Rev 2017;276:121-44.

16. Leone RD, Emens LA. Targeting adenosine for cancer immunotherapy. J Immunother Cancer 2018;6:57.

17. Syn N, Wang L, Sethi G, et al. Exosome-Mediated Metastasis: From Epithelial-Mesenchymal Transition to Escape from Immunosurveillance. Trends Pharmacol Sci 
2016;37:606-17.

18. Zhang H, Vijayan D, Li XY, et al. The role of NK cells and CD39 in the immunological control of tumor metastases. Oncoimmunology 2019;8:e1593809.

19. Simoni Y, Becht E, Fehlings M, et al. Bystander CD8(+) $T$ cells are abundant and phenotypically distinct in human tumour infiltrates. Nature 2018;557:575-9.

20. Yost KE, Satpathy AT, Wells DK, et al. Clonal replacement of tumor-specific T cells following PD-1 blockade. Nat Med 2019;25:1251-9.

21. Duhen T, Duhen R, Montler R, et al. Co-expression of CD39 and CD103 identifies tumor-reactive CD8 T cells in human solid tumors. Nat Commun 2018;9:2724.

22. Sade-Feldman M, Yizhak K, Bjorgaard SL, et al. Defining T Cell States Associated with Response to Checkpoint Immunotherapy in Melanoma. Cell 2018;175:998-1013.e20.

23. Li J, Wang L, Chen X, et al. CD39/CD73 upregulation on myeloid-derived suppressor cells via TGF- $\beta$-mTORHIF-1 signaling in patients with non-small cell lung cancer. Oncoimmunology 2017;6:e1320011.

24. Tøndell A, Wahl SGF, Sponaas A-M, et al. Ectonucleotidase CD39 and Checkpoint Signalling Receptor Programmed Death 1 are Highly Elevated in Intratumoral Immune Cells in Non-small-cell Lung Cancer. Transl Oncol 2020;13:17-24.

25. Li J, Wang L, Chen X, et al. CD39/CD73 upregulation on myeloid-derived suppressor cells via TGF-beta-mTORHIF-1 signaling in patients with non-small cell lung cancer. Oncoimmunology 2017;6:e1320011.

26. Oser MG, Niederst MJ, Sequist LV, et al. Transformation from non-small-cell lung cancer to small-cell lung cancer: molecular drivers and cells of origin. Lancet Oncol 2015;16:e165-72.

27. Goldstraw P, Chansky K, Crowley J, et al. The IASLC Lung Cancer Staging Project: Proposals for Revision of the TNM Stage Groupings in the Forthcoming (Eighth) Edition of the TNM Classification for Lung Cancer. J Thorac Oncol 2016;11:39-51.

28. Sadeghipour A, Babaheidarian P. Making FormalinFixed, Paraffin Embedded Blocks. Methods Mol Biol 2019;1897:253-68.

29. He Y, Yu H, Rozeboom L, et al. LAG-3 Protein Expression in Non-Small Cell Lung Cancer and Its Relationship with PD-1/PD-L1 and Tumor-Infiltrating Lymphocytes. J Thorac Oncol 2017;12:814-23.

30. Rohaan MW, van den Berg JH, Kvistborg P, et al. Adoptive transfer of tumor-infiltrating lymphocytes in melanoma: a viable treatment option. J Immunother Cancer 2018;6:102.

31. SPSS Statistics 22.0 Available online: https://www.ibm. com/support/pages/node/230551

32. He Y, Yu H, Rozeboom L, et al. LAG-3 Protein Expression in Non-Small Cell Lung Cancer and Its Relationship with PD-1/PD-L1 and Tumor-Infiltrating Lymphocytes. J Thorac Oncol 2017;12:814-23.

33. Graphpad Prism8. Available online: https://www.graphpad. com/

34. Wang S, Yang L, Ci B, et al. Development and Validation of a Nomogram Prognostic Model for SCLC Patients. J Thorac Oncol 2018;13:1338-48.

35. Früh M, De Ruysscher D, Popat S, et al. Small-cell lung cancer (SCLC): ESMO Clinical Practice Guidelines for diagnosis, treatment and follow-up. Ann Oncol 2013;24 Suppl 6:vi99-105.

36. Nicholson AG, Chansky K, Crowley J, et al. The International Association for the Study of Lung Cancer Lung Cancer Staging Project: Proposals for the Revision of the Clinical and Pathologic Staging of Small Cell Lung Cancer in the Forthcoming Eighth Edition of the TNM Classification for Lung Cancer. J Thorac Oncol 2016;11:300-11.

37. Ready N, Farago AF, de Braud F, et al. Third-Line Nivolumab Monotherapy in Recurrent SCLC: CheckMate 032. J Thorac Oncol 2019;14:237-44.

38. Antonia SJ, Lopez-Martin JA, Bendell J, et al. Nivolumab alone and nivolumab plus ipilimumab in recurrent smallcell lung cancer (CheckMate 032): a multicentre, openlabel, phase 1/2 trial. Lancet Oncol 2016;17:883-95.

39. Ott PA, Elez E, Hiret S, et al. Pembrolizumab in Patients With Extensive-Stage Small-Cell Lung Cancer: Results From the Phase Ib KEYNOTE-028 Study. J Clin Oncol 2017;35:3823-9.

40. Horn L, Mansfield AS, Szczesna A, et al. First-Line Atezolizumab plus Chemotherapy in Extensive-Stage Small-Cell Lung Cancer. N Engl J Med 2018;379:2220-9.

41. Paz-Ares L, Dvorkin M, Chen Y, et al. Durvalumab plus platinum-etoposide versus platinum-etoposide in firstline treatment of extensive-stage small-cell lung cancer (CASPIAN): a randomised, controlled, open-label, phase 3 trial. Lancet 2019;394:1929-39.

42. Takenaka MC, Gabriely G, Rothhammer V, et al. Control of tumor-associated macrophages and $\mathrm{T}$ cells in glioblastoma via AHR and CD39. Nat Neurosci 2019;22:729-40.

43. Li XY, Moesta AK, Xiao C, et al. Targeting CD39 in 
Cancer Reveals an Extracellular ATP- and InflammasomeDriven Tumor Immunity. Cancer Discov 2019;9:1754-73.

44. Yan J, Li XY, Roman Aguilera A, et al. Control of Metastases via Myeloid CD39 and NK Cell Effector Function. Cancer Immunol Res 2020;8:356-67.

45. Ahlmanner F, Sundstrom P, Akeus P, et al. CD39(+) regulatory $\mathrm{T}$ cells accumulate in colon adenocarcinomas and display markers of increased suppressive function. Oncotarget 2018;9:36993-7007.

46. Syed Khaja AS, Toor SM, El Salhat H, et al. Intratumoral FoxP3(+)Helios(+) Regulatory T Cells Upregulating Immunosuppressive Molecules Are Expanded in Human Colorectal Cancer. Front Immunol 2017;8:619.

47. Canale FP, Ramello MC, Nunez N, et al. CD39 Expression Defines Cell Exhaustion in Tumor-Infiltrating CD8(+) T Cells. Cancer Res 2018;78:115-28.

48. Sequeira I, Neves JF, Carrero D, et al. Immunomodulatory role of Keratin 76 in oral and gastric cancer. Nat Commun 2018;9:3437.

49. Limagne E, Euvrard R, Thibaudin M, et al. Accumulation of MDSC and Th17 Cells in Patients with Metastatic Colorectal Cancer Predicts the Efficacy of a FOLFOXBevacizumab Drug Treatment Regimen. Cancer Res

Cite this article as: Chen S, Wu S, Zhang L, Zhang W, Liu Y, Chen B, Zhao S, Li W, Sun C, Wang L, Jia K, Wang H, Chen P, Wu C, Zhu J, He Y, Zhou C. CD39: the potential target in small cell lung cancer. Transl Lung Cancer Res 2020;9(4):14831495. doi: 10.21037/tlcr-20-798
2016;76:5241-52.

50. Rao S, Yang H, Penninger JM, et al. Autophagy in non-small cell lung carcinogenesis: A positive regulator of antitumor immunosurveillance. Autophagy 2014;10:529-31.

51. Li JY, Duan XF, Wang LP, et al. Selective depletion of regulatory $T$ cell subsets by docetaxel treatment in patients with nonsmall cell lung cancer. J Immunol Res 2014;2014:286170.

52. Gabrielson A, Wu Y, Wang H, et al. Intratumoral CD3 and CD8 T-cell Densities Associated with Relapse-Free Survival in HCC. Cancer Immunol Res 2016;4:419-30.

53. Carvajal-Hausdorf D, Altan M, Velcheti V, et al. Expression and clinical significance of PD-L1, B7-H3, B7H4 and TILs in human small cell lung Cancer (SCLC). J Immunother Cancer 2019;7:65.

54. Yoon HH, Shi Q, Heying EN, et al. Intertumoral Heterogeneity of CD3(+) and CD8(+) T-Cell Densities in the Microenvironment of DNA Mismatch-RepairDeficient Colon Cancers: Implications for Prognosis. Clin Cancer Res 2019;25:125-33.

(English Language Editors: J. Jones and J. Chapnick) 
Supplementary

Table S1 Relationships between CD39 expression on TILs and other checkpoints (logistic regression analysis)

\begin{tabular}{lcccc}
\hline Characteristic & $\begin{array}{c}\text { Regression } \\
\text { coefficient }\end{array}$ & Standard error & Odds ratio & 95\% Cl \\
\hline PD-L1 expression on TILs & 1.569 & 0.573 & 4.800 & $1.561-14.764$ \\
CD3 expression on TILs & 2.610 & 0.595 & 13.600 & $4.234-43.680$ \\
CD4 expression on TILs & 3.595 & 1.060 & 36.400 & $4.556-290.808$ \\
CD8 expression on TILs & 21.351 & $8,770.825$ & $1.9 \times 10^{9}$ & -0.001 \\
FOXP3 expression on TILs & 3.332 & 1.060 & 28.000 & $3.510-233.387$ \\
\hline
\end{tabular}

PD-L1, programmed cell death-ligand 1; FOXP3: forkhead box P3; TIL, tumor-infiltrating lymphocyte. 
Table S2 Predictive factors of RFS in SCLC

\begin{tabular}{|c|c|c|c|c|c|c|c|}
\hline \multirow[t]{2}{*}{ Characteristic } & \multicolumn{2}{|c|}{ Log-rank (Mantel-Cox) test } & \multicolumn{3}{|c|}{ Median survival } & \multicolumn{2}{|c|}{$\begin{array}{c}\text { Hazard ratio (log-rank) (negative } \\
\text { vs. positive) }\end{array}$} \\
\hline & Chi square & $P$ value & Value & Ratio & $95 \% \mathrm{Cl}$ & Ratio & $95 \% \mathrm{Cl}$ \\
\hline$<70$ & 6.328 & 0.0119 & 38.10 & 2.610 & $1.376-4.950$ & 0.4245 & $0.1964-0.9172$ \\
\hline Female & 1.430 & 0.2318 & Undefined & Undefined & Undefined & 0.5742 & $0.2643-1.247$ \\
\hline Male & & & 28.50 & & & & \\
\hline \multicolumn{8}{|c|}{ Smoking status, n (\%) } \\
\hline Stage I-II & 7.890 & 0.0050 & 63.00 & 4.038 & $2.109-7.734$ & 0.4087 & $0.2186-0.7640$ \\
\hline Stage III-IV & & & 15.60 & & & & \\
\hline \multicolumn{8}{|l|}{ Chemotherapy } \\
\hline No & 0.9663 & 0.3256 & 15.10 & 0.3963 & $0.2346-0.6694$ & 1.295 & $0.7613-2.202$ \\
\hline Yes & & & 38.10 & & & & \\
\hline \multicolumn{8}{|c|}{ CD39 expression on TILs } \\
\hline Negative & 4.259 & 0.0390 & 45.00 & 2.500 & $1.279-4.887$ & 0.5222 & $0.2765-0.9862$ \\
\hline Negative & 4.487 & 0.0342 & 15.20 & 0.3680 & $0.1883-0.7193$ & 2.003 & $1.037-3.868$ \\
\hline Positive & & & 41.30 & & & & \\
\hline \multicolumn{8}{|c|}{ CD4 expression on TILs } \\
\hline Negative & 4.364 & 0.0367 & 16.20 & Undefined & Undefined & 2.166 & $1.125-4.169$ \\
\hline Positive & & & Undefined & & & & \\
\hline \multicolumn{8}{|c|}{ CD8 expression on TILs } \\
\hline Negative & 3.851 & 0.0497 & 16.20 & Undefined & Undefined & 2.286 & $1.136-4.597$ \\
\hline Positive & & & Undefined & & & & \\
\hline \multicolumn{8}{|c|}{ FOXP3 expression on TILs } \\
\hline Negative & 4.058 & 0.0440 & 17.00 & Undefined & Undefined & 2.230 & $1.137-4.374$ \\
\hline Positive & & & Undefined & & & & \\
\hline
\end{tabular}

RFS, relapse-free survival; Cl, confidence interval; SCLC, small cell lung cancer; TIL, tumor-infiltrating lymphocyte; PD-L1, programmed cell death-ligand 1; FOXP3, forkhead box P3. 
Table S3 Univariate and multivariate COX regression analysis of RFS

\begin{tabular}{|c|c|c|c|c|c|c|}
\hline Variables & \multicolumn{3}{|c|}{ Univariate } & \multicolumn{3}{|c|}{ Multivariate } \\
\hline Age (<70 vs. $\geq 70)$ & 2.413 & $1.257-4.632$ & 0.008 & 1.922 & $0.944-3.913$ & 0.072 \\
\hline Gender (female vs. male) & 1.767 & $0.685-4.562$ & 0.239 & & & \\
\hline Smoking status (Non-smoker vs. smoker) & 1.297 & $0.990-2.896$ & 0.428 & & & \\
\hline Chemotherapy (negative vs. positive) & 0.821 & $0.431-1.566$ & 0.550 & & & \\
\hline CD39 expression on TILs (negative vs. positive) & 2.161 & $1.019-4.580$ & 0.044 & 3.962 & $1.632-9.617$ & 0.002 \\
\hline PD-L1 on TILs (negative vs. positive) & 0.436 & $0.205-0.925$ & 0.030 & 0.510 & $0.172-1.516$ & 0.226 \\
\hline CD3 expression on TILs (negative vs. positive) & 0.483 & $0.242-0.963$ & 0.039 & 0.692 & $0.236-2.028$ & 0.503 \\
\hline FOXP3 expression on TILs (negative vs. positive) & 0.439 & $1.370-5.153$ & 0.051 & & & \\
\hline
\end{tabular}

RFS, relapse-free survival; CI, confidence interval; SCLC, small cell lung cancer; TIL, tumor-infiltrating lymphocyte; PD-L1, programmed cell death-ligand 1; FOXP3, forkhead box P3. 